\title{
A Study of Multi-channel Wavelength Division Multiplexing Using Photonic Crystals
}

\author{
M. H. Chen ${ }^{1}$, C. F. Chang ${ }^{1}$, Y. D. Wu ${ }^{2}$ and S. Y. Chen ${ }^{1}$ \\ ${ }^{1}$ Institute of Electrical Engineering National Sun Yat-Sen University, Kaohsiung, \\ 804, Taiwan, R.O.C. \\ ${ }^{2}$ Electronic Engineering of National Kaohsiung University of Applied Sciences, Kaohsiung, 807, Taiwan, R. O. C. ${ }^{2}$ \\ Phone: 886-7-5252000 \#4166 \\ E-Mail: p923010020@student.nsysu.edu.tw
}

\begin{abstract}
This paper demonstrated a multi-channel Wavelength-Division-Multiplexing (WDM) system using photonic crystal structures. The system consists of a waveguide that realized by a defect row of photonic crystal and high Q-value micro-cavities. Two-Dimension (2-D) Finite-Difference-TimeDomain (FDTD) method is performed for simulation in this paper. The results showed good ability to filter an incident pulse into six spectral channels with a FHWM of $1.4 \mathrm{~nm}$ at center wavelength $1550 \mathrm{~nm}$ output channel. Six-channel coarse wavelength division multiplexer (CWDM) from 1490 1590nm with channel spacing of $20 \mathrm{~nm}$ which defined by ITU-T Recommendation G.694.2 are presented.
\end{abstract}

Keywords: Photonic crystal, FDTD, WDM, CWDM.

\section{Introduction}

Photonic crystals (PCs) have become the worldwide interesting studies recently. Studies focus on high efficiency of transmission rate to maintain light propagation in photonic crystal structure. This idea periodic dielectric structure can possess the property of band gap for certain regions in the frequency spectrum [1, 2]. In the micro-cavity with waveguide application, the possibilities to implement optical filter in PCs base into multi-channel add-drop filter $[3,4]$ is proposed. In wavelength division multiplexing application, multi-channel with an incident wavelength $1 \mu \mathrm{m}$ is proposed [5]. For higher drop efficiency improve of photonic crystal-based multichannel drop filter, Sangin Kim [6] proposed a reflection feedback system and Honglian Ren [7] proposed a wavelength-selective reflection microcavity system. These optoelectronic devices, which are designed by photonic crystal technology, are micrometers scale. The compact size of optoelectronic devices in PCs base can be fabricated by semiconductor process technology and easy to be realization by current process.

In communication system or fiber optic sensors application field, WDM system is useful for better bandwidth utilization of multiplexing technology. Currently, Coarse-Wavelength-Division-Multiplexing (CWDM) and Dense-Wavelength-DivisionMultiplexing (DWDM) systems connect with optical fiber interferometers system are very important for fiber optic sensors application. In this kinds of system, three conventional optical interferometers Michelson type, Mach-Zehnder type and Sagnac type are widely using. Only Sagnac type is not concern with coherence length ( $\mathrm{L}_{\mathrm{C}}=-\lambda^{2} / \Delta \lambda, \lambda$ is light source wavelength) in optical sensing system loop. CWDM system channel spacing is standardized at 20nm which is wide enough to accumulate wavelength and has more cost effectiveness with inexpensive and broadband laser light source using than DWDM system.

Recently, such a WDM system based on channel drop filters with reflector to improve drop efficiency have been proposed by [6, 7] groups. In their structures, one [6] is suffered lower coherence length by $5 \times 5$ super-cells with end of the bus waveguide reflector design, and the other [7] needs large area to design highly efficiency add-drop filter pairs which need five lattice constant distances of two microcavities for best drop efficiency request. This paper proposed a new approach of six-channel CWDM in PCs which has large coherence length and keep higher transmission ratio by asymmetry super cell structure micro-cavities (7x7 super-cell like) and wave reflectors design. Two-Dimension (2-D) FiniteDifference-Time-Domain (FDTD) method is performed for simulation and the results showed good ability to improve coherence length and without transmission ratio degradation. The results showed good ability to filter the incident pulse into six spectral channels of center wavelength from 1490 1570nm 
with spacing 20nm and coherence length $1.716 \mathrm{~cm}$ (FHWM 1.4nm) at center wavelength 1550nm output channel. The compact (micrometer scale) and simple structure is useful to integrate optics circuit realization and future device fabrication.

\section{Design and Simulation Result}

In this paper, high refractive index pillars in air on square array structure in PCs structure are presented. The refractive index of $\operatorname{In}_{0.53} \mathrm{Al}_{0.16} \mathrm{Ga}_{0.31}$ As rod is 3.19 for an incident wavelength $1.55 \mu \mathrm{m}$ is selected. 2-D FDTD method is employed to calculate the photonic band gap. The maximum band gap is gotten with (r/a) ratio 0.185 (rod radius: 101nm; lattice constant: $546 \mathrm{~nm}$ ), the normalize frequency of the photonic band gap is between 0.32 and 0.45 which is shown in Fig.1. The point defect of refractive index 2.645 and different radius $\left(r_{1}=51.63 \mathrm{~nm}, r_{2}=55.92 \mathrm{~nm}, r_{3}=\right.$ $\left.60.21 \mathrm{~nm}, r_{4}=64.51 \mathrm{~nm}, \quad r_{5}=68.80 \mathrm{~nm}, \quad r_{6}=73.09 \mathrm{~nm}\right)$ which is shown in Fig.2 is selected, respectively.

As Fig.2 is shown, for transmission ratio and band width improvement, we proposed asymmetry super cell micro-cavities with point defects to get higher NXN super-cell with narrow defect band width performance and use wave reflectors in the waveguides system to enhance the transmission ratio. The simulation result showed the system has good ability to filter an incident light pulse into six-channel spectral frequency with FHWM $1.4 \mathrm{~nm}$ (coherence length $1.716 \mathrm{~cm}$ ) at center wavelength $1550 \mathrm{~nm}$ output channel. As the result is shown in Fig.3, six-channel output wavelengths are $\lambda_{1}=1492 \mathrm{~nm}, \lambda_{2}=1510 \mathrm{~nm}$, $\lambda_{3}=1529 \mathrm{~nm}, \lambda_{4}=1550 \mathrm{~nm}, \lambda_{5}=1570 \mathrm{~nm}$ and $\lambda_{6}=1592$ $\mathrm{nm}$, respectively. As the results are shown in Fig. 4-9, the system is successful to separate the different incident wavelengths to different output channels. The wavelength division multiplexing filters the light with various frequencies exactly is presented in this paper. The device design is lead the way to achieve CWDM which defined by ITU-T Recommendation G.694.2 and has good capability to extend the application of fiber optical sensor field.

\section{Conclusion}

As the result, good ability of six-channel wavelength division multiplexing and achieve CWDM which defined by ITU-T Recommendation G.694.2 are presented. The compact and simple structure in PCs base is the advantage of WDM application. Consequently, such kinds of device will be more useful to integrate optics circuit realization and future device fabrication.

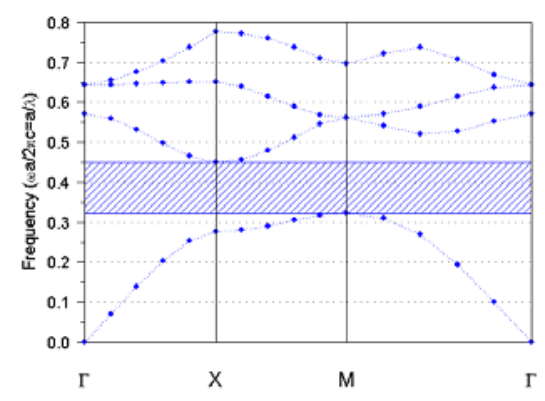

Fig.1 Band structure of photonic crystal structure

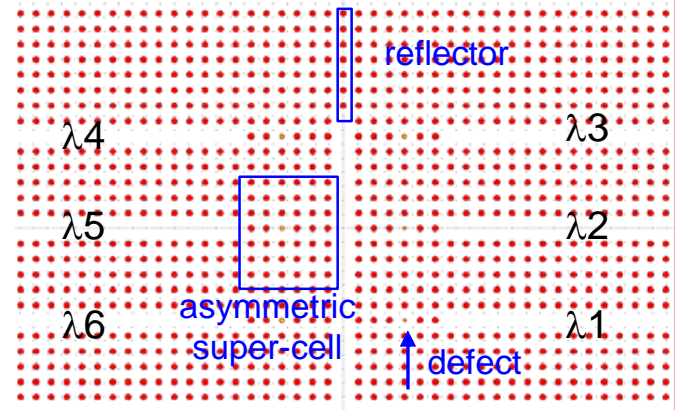

Fig.2 2-D six-channel asymmetry super cell micro-cavity with reflector photonic crystal WDM structure

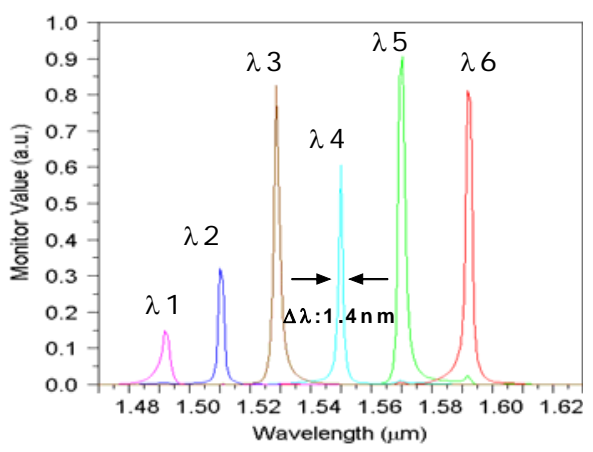

Fig.3 Asymmetry array micro-cavity with reflector output spectral channel wavelength and FHWM $\sim 1.4 \mathrm{~nm}$ at $1550 \mathrm{~nm}$ channel $\left(\lambda_{1}: 1492 \mathrm{~nm} ; \lambda_{2}: 1510 \mathrm{~nm} ; \lambda_{3}: 1529 \mathrm{~nm} ; \lambda_{4}: 1550 \mathrm{~nm}\right.$; $\left.\lambda_{5}: 1.570 \mathrm{~nm} ; \lambda_{6}: 1592 \mathrm{~nm}\right)$ 

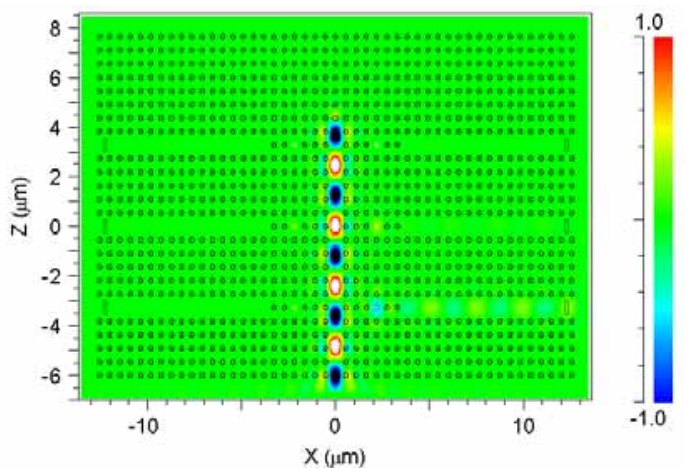

Fig.4 The output channel 1 of incident wavelength 1490nm
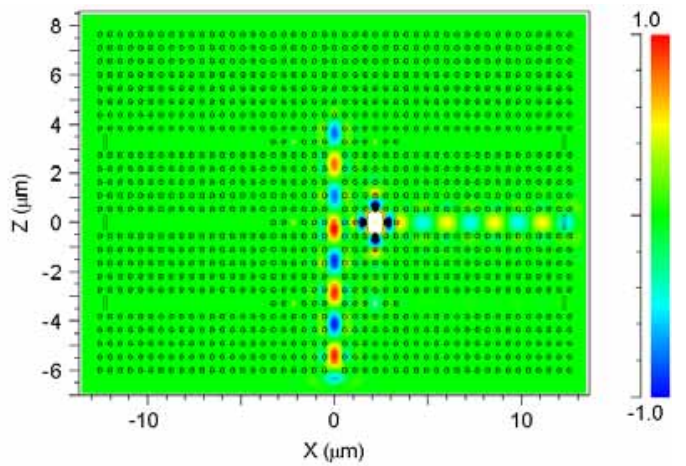

Fig.5 The output channel 2 of incident wavelength 1510nm
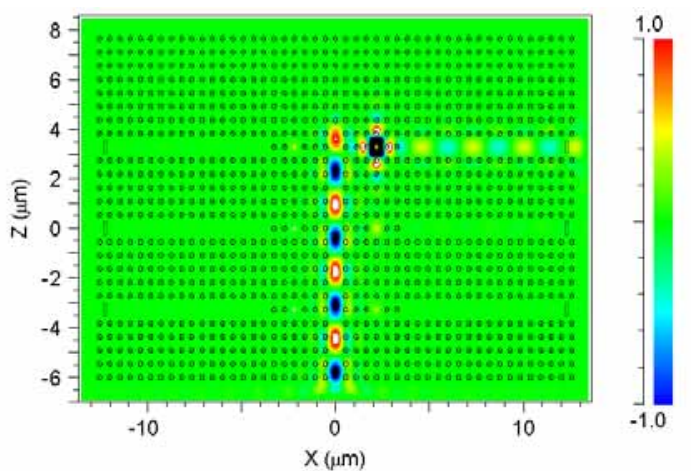

Fig.6 The output channel 3 of incident wavelength 1530nm

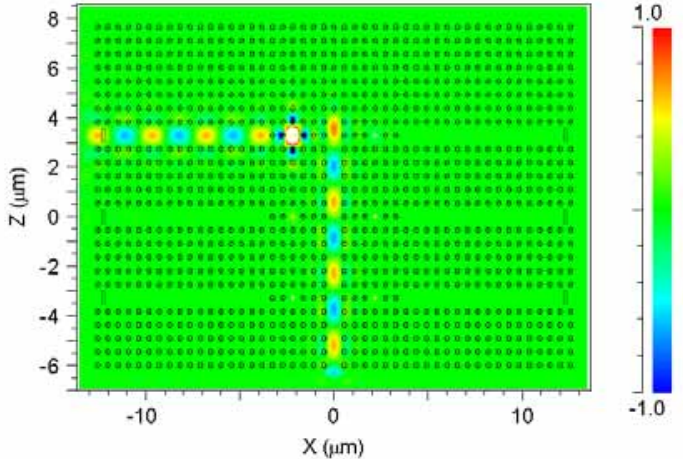

Fig.7 The output channel 4 of incident wavelength $1550 \mathrm{~nm}$

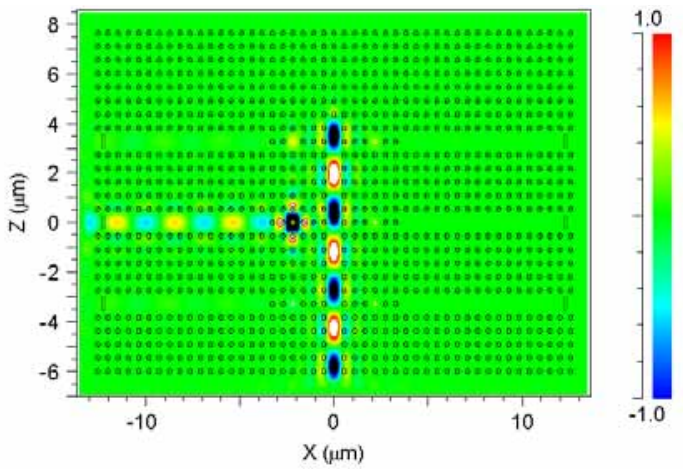

Fig.8 The output channel 5 of incident wavelength $1570 \mathrm{~nm}$

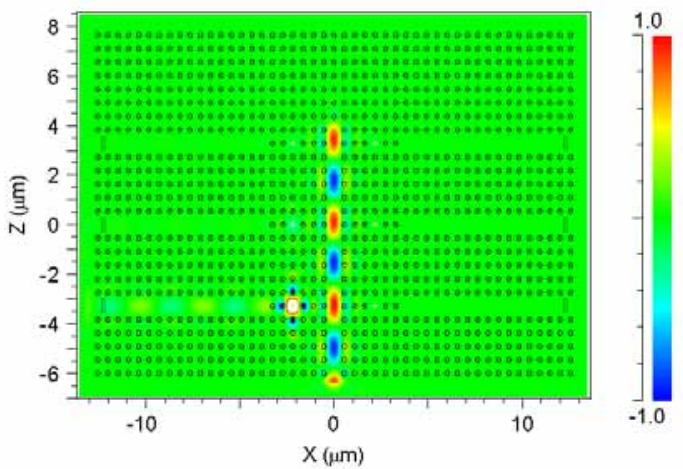

Fig.9 The output channel 6 of incident wavelength 1590nm 


\section{References}

[1] E. Yablnovitch, "Inhibited spontaneous emission in solid-state physics and electronics," Phys. Rev. Lett. 58, pp. 2059-2062, 1987.

[2] S. John, "Strong localization of photons in certain disordered dielectric superlattices," Phys. Rev. Lett. 58, pp. 2486-2489, 1987.

[3] Shanhui Fan, P. R. Villeneuve, J. D. Joannopoulos, "Channel drop filters in photonic crystals," Optics Express, Vol. 3, pp. 4-11, 1998.

[4] Bong-Shik Song, Takashi Asano, Yoshihiro Akahane, Yoshinori Tanaka, and Susumu Noda, "Multichannel Add/Drop Filter Based on In-Plane Hetero Photonic Crystals," Journal of Lightwave Technology, Vol. 23, pp. 1449-1455, 2005.

[5] Ahmed Sharkawy, Shouyuan Shi, and Dennis W. Pratrher, "Multichannel wavelength division multiplexing with photonic crystals," Applied Optics, Vol. 40, pp. 2247-2252, 2001.

[6] Sangin Kim, Ikmo park, Hanjo Lim, and Chul-Sik Kee, "Highly efficient photonic crystal-based multi-channel drop filters of three-port system with reflection feedback," Optics Express, Vol. 12, pp. 5518-5525, 2004.

[7] Honglian Ren, Chun Jian, Weisheng Hu, Mingyi Gao, Jingyuan Gao and Jingyuan Wangm, "Photonic crystal channel drop filter with a wavelength-selective reflection micro-cavity," Optics Express, Vol. 14, pp. 2446-2458, 2006. 